\title{
Mandibular Residual Alveolar Bone Height Changes in Two Different Design Concepts of Four Implant Assisted Overdenture
}

\author{
Doaa Elmowafy ${ }^{1}$, Mohamed M. Fouad ${ }^{2}$,Nessreen Elmekkawy ${ }^{3}$ and Mohamed Elgamal ${ }^{4}$ \\ ${ }^{1}$ Assistant lecturer at prosthodontic department, Mansoura University \\ ${ }^{2}$ Professor of prosthodontics, Mansoura University \\ ${ }^{3}$ Professor of prosthodontics, Mansoura University \\ ${ }^{4}$ lecturer of prosthodontics, Mansoura University
}

\begin{abstract}
:
Purpose: This comparative study was conducted to compare between two different design concepts of four implant assisted complete mandibular overdenture regarding the residual alveolar bone height changes.

Materials and Methods: Ten healthy completely edentulous male patients were selected for this study. For each patient, a conventional complete denture was constructed. Each mandibular denture was duplicated to be used as a radiographic stent, then the CBCT surgical guide template was printed according to each design concept of 4-implant overdenture. The CBCT surgical guide templates were used for installing the implants according to flapless surgical technique followed by delayed loading. According to the two design concepts of the 4-implant overdenture used in this study, the patients were randomly classified into two equal groups: group [A] had two vertical canine implants with two axial 1st molar short implants (short design concept), and group [B]had two vertical canine implants with two distally inclined premolar implants (inclined design concept). Radiographic evaluations were performed immediately (T0), 6 months (T6), and 12 months (T12) respectively after picking up of the ball attachments.

Results: the residual bone resorption in the two groups after one year of loading was within the accepted limits (Group A $0.56 \pm 0.4 \mathrm{~mm}$, Group B $0.67 \pm 0.03 \mathrm{~mm}$ ). Also, these results showed a significant decrease in bone resorption in both groups during the 2 nd 6 month after loading. A statistically significant higher ridge resorption was found in group $B$ during the first six months $(p=0.006)$ whereas in the second six months, the difference was insignificant.

Conclusion: From the results of this study, it could be concluded that: 1- In regard to the accepted limits of residual alveolar bone loss, the two design concepts with either 1st molar short implant or premolar distally inclined implant can be used for assisting the mandibular complete overdentures. 2- The posterior short implant concept can be considered more favorable design than inclined implant concept when designing the 4-implant assisted mandibular complete overdenture, regarding the preservation of alveolar bone height.
\end{abstract}

Key words: Short implant, inclined implant, ball attachment, 4-implant overdenture.

\section{Introduction:}

Conventional complete dentures have been the standard option of care for more than a century. Complete denture wearers are usually satisfied with the upper denture but the majority of them often struggle to eat with the lower denture because of the lack of retention ${ }^{[1,2]}$.

With the aid of dental implant, alternative treatment options can be used for edentulous patient: implant assisted overdentures, and implant supported fixed dental prostheses (fixed dentures) ${ }^{[3]}$.

The number and distribution of loaded implants affect the supporting residual alveolar bone. The use of two implants to assist the mandibular complete overdentures is commonly accepted as alternative treatment 
option to complete denture, although it's problems of poor implant support and stability, mandibular ridge resorption distal to the implants due to stresses transmitted to the ridge as a result of rotation of the denture around the anterior implants and increase the rate of attachment wearing during function ${ }^{[4]}$.

Adding two posterior implants may increase the support of the denture and prevent its rotational movements. In the mandible, the inferior alveolar nerve and associated structures may provide minimal bone for implant anchorage or prevent the placement of implants distal to the mental foramina ${ }^{[5]}$.

Four implants have been used for anchoring mandibular overdentures as early as the mid-1980s. Multiple implants for mandibular overdenture are indicated when sensitive jaw anatomy, increased occlusal forces, or high retention requirement are present or when narrow diameter or short implant are employed. Four implants provide more stability during function, and avoid both movement and excessive loading, which may compromise osseointegration ${ }^{[6]}$.

In mandibular alveolar ridge, placement of standard-length implants is hindered by severely resorbed alveolar ridges due to anatomical vital structures limitations such as the mental foramen, inferior alveolar nerve, mandibular canal and shape of the ridge. To avoid grafting procedures and to utilize preexisting bone in the most effective way, short implants have been proposed also as an alternative to the long ones. In addition, they also reduce the morbidity, treatment time, costs and complications rates ${ }^{[7]}$.

Tilting of the implants may be a viable possible treatment option. This technique includes surgical and prosthetic advantages, such as the possibility of placing long implants with enhancement of bone anchorage, the decrease of the requirement for bone grafting, avoiding long cantilevers and the possibility of increasing the distance between anterior and posterior abutments with enhancement of the load distribution ${ }^{[8]}$.

Another treatment option is short implant. Short implant is simple, cost effective and highly predictable. In particular, practitioners want to rehabilitate most patients without implementing advanced surgical techniques, such as alveolar augmentations Placement of short implants less than $8 \mathrm{~mm}$ long may be considered an effective option to rehabilitate edentulous patients whenever conventional implants cannot be placed without prior bone augmentation procedures, such as bone graft, osteogenic distraction or mental foramen transposition, which increase surgical morbidity and treatment time ${ }^{[9]}$.

Therefore, the purpose of this study is to evaluate mandibular residual alveolar bone height changes when using two axial canine implants with either two short first molar implants or two premolar inclined implants for assisting mandibular complete overdenture.

\section{Material and Methods:}

Ten healthy completely edentulous male patients (aged between 52-64 years with mean age 58 years) were selected from the outpatient clinic of Prosthodontics Department according to the following criteria: all patients have sufficient mandibular residual alveolar ridges bone of type D3 at least verified by C.B.C.T, one year at least after last extraction, covered with even thickness, firm healthy mucosa, with no previous denture history, Angle's class I maxillomandibular relation, adequate restorative space verified by putty index method. Exclusive criteria were patients with history of parafuncional habits, smoking, alcohol administration, systematic disorders affecting bone as diabetes, history of radiotherapy in the head and neck region, TMJ or neuromuscular disorders.

. For each patient, conventional complete denture was constructed, inserted and the patients were instructed to wear the dentures for one month before implantation with weekly follow up visits till no complaint. The stereolithographic surgical guide was constructed to determine the exact location, parallelism and inclination of the implants. According to the 4-implant design concept, the patients were randomly classified in to two equal groups as follow:

- Group A: two vertical standard implants $(3.7 * 13 \mathrm{~mm})$ were inserted in canine region and two vertical short implants $\left(4.3^{*} 6 \mathrm{~mm}\right)$ were inserted in the $1^{\text {st }}$ molar areas.

- Group B: two vertical standard implants $\left(3.7^{*} 13 \mathrm{~mm}\right)$ were inserted in canine region and two $30^{\circ}$ distally inclined implants $(3.7 * 13 \mathrm{~mm})$ were inserted in the $1^{\text {st }}$ premolar areas. 
- The four implants were inserted according to the flapless surgical technique followed by delayed implant loading protocol. After three months of osseointegration period, a second stage surgery was done and healing abutments will be secured into implants for two weeks. Then healing abutments were replaced by ball attachments. Ball attachments with 2-3 mm gingival height were screwed in the parallel fixtures. For group $\mathrm{B}, 30^{\circ}$ angled ball attachments were used to be parallel with the axial attachments (composed of multiunit abutment with $30^{\circ}$ angulation screwed firstly in the fixture then screwing the ball over the abutment). Relief in the site of the female housing of the ball attachment and Small vents were made lingual to the prepared cavities for easy escape of the excess resin. The female housings were picked up using autopolymerized acrylic resin while the patient closed in centric occlusion. The denture was removed, finished and polished. Intraoral readjustment of occlusion was done. The patient was instructed of oral hygiene measures. fig $(1,2)$

- Radiographic evaluations were done immediately (T0), six (T6) and 12 (T12) months after insertion of definitive overdenture.

- Statistical Analysis was made. The SPSS statistical package for social science version 22 (SPSS Inc., Chicago, IL, USA) ${ }^{[10]}$. was used for data analysis. Shapiro-Wilk test was used to test the normality of the bone loss values. The data was parametric and normally distributed. Descriptive statistics were performed in terms of mean, median, standard deviation, range, minimum, maximum. Repeated measures ANOVA was used to test significant difference in bone loss between time intervals followed by Bonferroni post hoc test for multiple comparison between each time intervals. Independent samples t-test was used to compare bone loss between groups. $\mathrm{P}$ is significant if $<0.05$ at confidence interval $95 \%$.
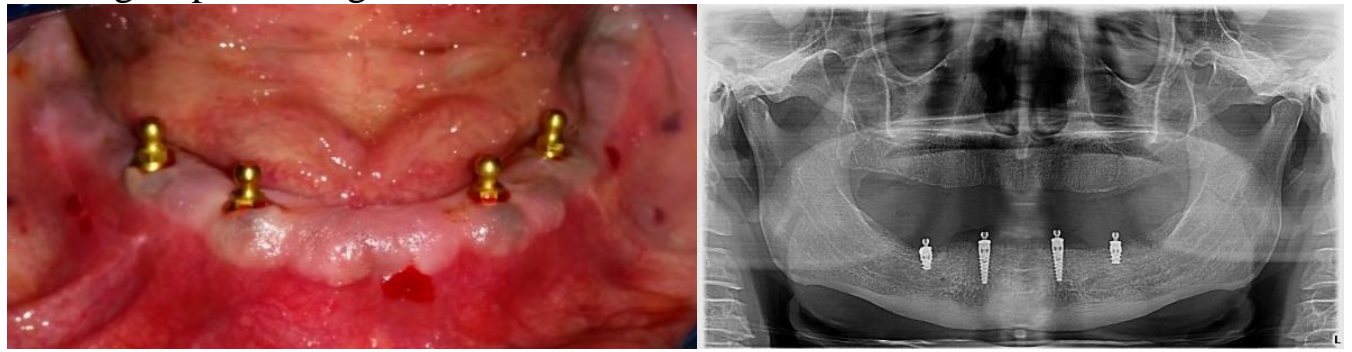

Fig 1: Implant fixture with their ball attachments in group A
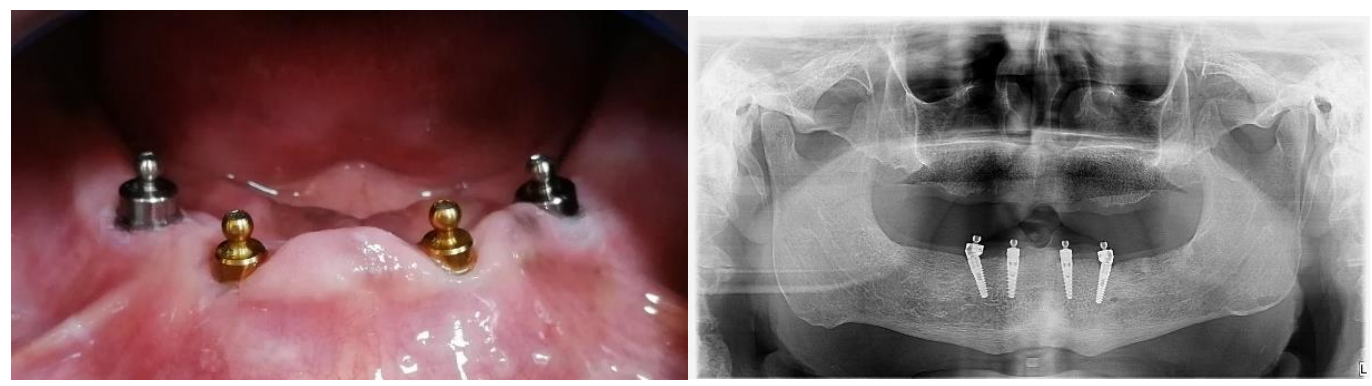

Fig 2: Implant fixture with their ball attachments in group B

\section{Results:}

- Comparison of bone loss between different time intervals for group A is presented in table 1.

- There was a significant difference between time intervals for group A (Repeated measure ANOVA, $\mathrm{p}<.001)$

- Multiple comparison between each 2 time intervals using Bonferroni post hoc test is presented in table 3

- There was a significant difference in bone loss between the first and the second 6 months after overdenture insertion (Bonferroni, $\mathrm{p}=.004$ ) 
- There was a significant difference in bone loss between the second 6 months after overdenture insertion and total bone loss after 12 months from insertion (Bonferroni, $\mathrm{p}=.001$ )

Table 1. Comparison of bone loss between different times intervals for group A

\begin{tabular}{|l|r|r|r|}
\hline & (T0-T6) & (T6-T12) & (T0-T12) \\
\hline Mean & .3300 & .2329 & .5629 \\
\hline Std. Deviation & .0405 & .0172 & .0412 \\
\hline F value & \multicolumn{3}{|c|}{767.344} \\
\hline $\begin{array}{c}\text { Repeated measures ANOVA (P } \\
\text { value) }\end{array}$ & $.004^{*}$ & $.001^{*}$ & $<.001^{*}$ \\
\hline $\begin{array}{c}\text { Bonferroni post-hoc test (p } \\
\text { value) }\end{array}$ & & & \\
\hline
\end{tabular}

- Comparison of bone loss between different time intervals for group B is presented in table 2.

- There was a significant difference between time intervals for group B (Repeated measure ANOVA, $\mathrm{p}<.001)$

- There was a significant difference in bone loss between the first and the second 6 months after overdenture insertion (Bonferroni, $\mathrm{p}=.001$ )

- There was a significant difference in bone loss between the second 6 months after overdenture insertion and total bone loss after 12 months from insertion (Bonferroni, $\mathrm{p}<.001$ )

Table 2. Comparison of bone loss between different times intervals for group B

\begin{tabular}{|l|c|c|c|}
\hline & (T0-T6) & (T6-T12) & (T0-T12) \\
\hline Mean & .4300 & .2414 & .6714 \\
\hline Std. Deviation & .0447 & .0205 & .0364 \\
\hline F value & 980.781 & \\
\hline $\begin{array}{l}\text { Repeated measures } \\
\text { ANOVA (P value) }\end{array}$ & $<.001^{*}$ & \multicolumn{2}{|l}{} \\
\hline $\begin{array}{l}\text { Bonferroni post-hoc } \\
\text { test } \\
\text { (p value) }\end{array}$ & $.001^{*}$ & $<001 *$ \\
\cline { 2 - 4 } & $<.001^{*}$ \\
\cline { 2 - 4 } &
\end{tabular}

\section{Comparison of bone loss between groups}

- Comparison of bone loss between groups at different observation times is presented in fig 3 .

- In the first 6 months after overdenture insertion, there was a significant difference in bone loss between groups (independent samples t-test, $\mathrm{p}=.006$ ). Group B showed significant higher bone loss than group A. 
- In the second 6 months after overdenture insertion, no significant difference in bone loss between groups was noted (independent samples t-test, $\mathrm{p}=.494$ )

- In the total bone loss from insertion to 12 months after insertion, there was a significant difference in bone loss between groups (independent samples t-test, $\mathrm{p}=.002$ ). Group B had significant higher bone loss than group A.

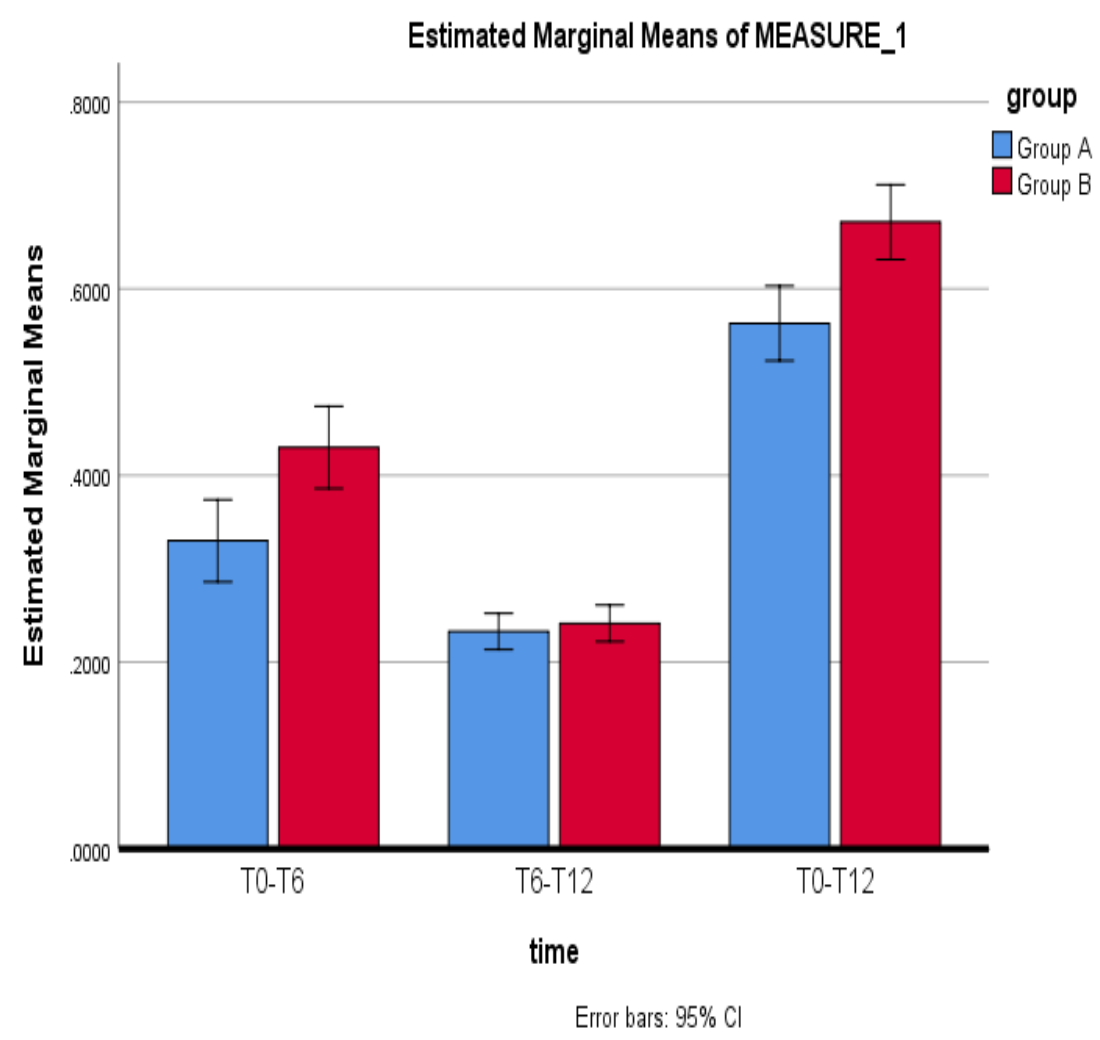

- Fig (3): Comparison of bone loss between groups at different time intervals

\section{Discussion:}

The result of this study showed that the residual bone resorption in the two groups after one year of loading was within the accepted limits (less than $0.67 \mathrm{~mm}$ ) which was lower than the rate of residual bone resorption in patients rehabilitated with complete denture $\left(1.6 \pm 0.1 \mathrm{~mm} /\right.$ year) as reported by Babu et al (2017) ${ }^{[11]}$.

In addition to increasing retention and support for prosthesis, another beneficial result of providing implant is the preservation of the existing residual bony ridge. Implant overdentures are intended to preserve the residual alveolar bone by maintaining the mechanical stimulation of bone tissue. ${ }^{[12]}$

Based on the findings of a clinical study by $\mathbf{S . K m}$ et al $\mathbf{2 0 2 1}{ }^{[13]}$, it can be concluded that ridge resorption is lesser in implant mandibular overdenture compared with conventional complete denture. Irrespective of the type of rehabilitation, posterior mandible is most vulnerable to ridge resorption. However, the extent of resorption is lesser in implant overdenture than complete denture in posterior mandible, thus implying implant has a protective or a shielding effect on posterior mandible from resorption.

Similarly, Jayaram and shenoy (2017) ${ }^{[14]}$ recommended early implant placement for implantsupported overdentures instead of the conventional complete denture therapy in a young patient as it is found that residual ridge resorption (RRR) increases with duration of edentulousness, so , attempts must be made to preserve the residual alveolar bone.

Kordatzis et al. ${ }^{[15]}$ investigated bone loss of the mandibles restored with implant overdentures (IOD) compared with either fixed prostheses or complete dentures and have reported less ridge resorption in posterior mandible rehabilitated with implant-supported overdenture $(0.69 \mathrm{~mm})$ when compared with rehabilitation with complete denture $(1.63 \mathrm{~mm})$ over a period of 5 years.

Additionally, in a study conducted by Wright et $\mathbf{a l}^{[16]}$, the results showed low rates of posterior mandibular residual ridge resorption for patients with advanced mandibular resorption who were 
rehabilitated with implant stabilized mandibular overdentures. It was suggested that dynamic loading of bone can have a profound osteogenic effect.

The same result was explained by Salleh et $\mathbf{a l}^{[17]}$ and Bae-JC et al $^{[18]}$ where there was lesser posterior ridge resorption with implant prostheses compared to conventional complete dentures, even though higher chewing forces and improved masticatory function were reported with implant therapy.

In addition to that, Kremer et al ${ }^{[19]}$ suggested that the distal flange of a mandibular implant overdenture may be a local factor that increases bone resorption, whereas implants seem to protect the neighboring bone from resorption.

The decrease of bone loss in both groups concurred with the results of Kordatzis et al. ${ }^{[15]}$ where they employed proportional area measurements using panoramic radiographs to determine ridge reduction within 5 years. They attributed this to the more favorable loading and protection of the residual posterior ridge from excessive loading by the mandibular anterior implants. Similarly, a recent systematic review conducted by Khalifa et al 2016 ${ }^{[20]}$ concluded alveolar ridge preservation with implant-assisted restorations.

Moreover, Emara (2019) ${ }^{[21]}$ stated that implant Mandibular overdentures retained by resilient attachment systems provide a low acceptable range of residual ridge resorption after 5years of function

It has been suggested that functional loading and light irritative stimuli could lead to changes in bone architecture, shape, and volume, and that by placing implants in the edentulous mandible and subsequently loading them, functional conditions could be created to limit bone resorption in the distal area of the mandibular alveolar ridge. ${ }^{[22]}$

The Mechanostat theory, first proposed by Frost in 1987 describes a regulatory mechanism within bone that deals with strain magnitude and invokes bone formation and resorption as the primary "effector mechanisms" by which bone alters its structure and properties in response to mechanical usage. ${ }^{[23]}$ Frost's theory suggests that bone can act like a mechanostat, in that it brings about a biomechanical adaptation, corresponding to the external loading condition. The theory proposed four microstrain zones, which correlated to a mechanical adaptation: (a) disuse atrophy; (b) steady state; (c) physiological overload; and (d) pathological loading. ${ }^{[24]}$

In contrast to this result, W. Adina et al (2015) ${ }^{[22]}$ studied the bone height changes in the posterior area of the mandible after implant placement and loading with an overdenture on two or four implants over a mean follow-up period of 10.5 years and no clinically relevant difference was found between the posterior mandible height before implant placement and at follow-up after functional loading with an implantsupported mandibular overdenture although the average mandibular bone height reduction of $0.5 \mathrm{~mm}$ was measured.

Also, Tymstra et al ${ }^{[25]}$ reported almost equal amount of ridge resorption in posterior mandible in implant overdenture compared to conventional complete denture over a period of 10 years.

When comparing between the bone loss during the time intervals, a significant decrease of residual alveolar bone loss was found in the second six months after implant overdenture insertion in both groups. This may be due to the gradual decrease of rotational movement of the overdenture due to more overdenture settlement with time.

This explanation is concurred with UTZ-KH ${ }^{[26]}$ that the denture settlement decreases gradually by time and result in decrease in the overdenture rotational movement which decreases stresses on the implants and the residual ridge.

Furthermore, the decrease of the bone loss during the $2^{\text {nd }} 6$ months after loading may be due to increasing the bone density because of an increase in mineral content which led to increasing strength of the bone from the beginning of loading after overdenture insertion and up to 1 year after loading according to Roberts et al. ${ }^{[27]}$ who described this phenomenon as a "steady state" and correlated the decrease of bone loss around dental implants at the end of first year to maturation of bone after implant placement and adaptation of bone to resist functional force.

The lesser extent of resorption in group A implant overdentures can be attributed to enhanced stability of dentures resulting in more favorable distribution of forces over the ridge. It can also be attributed to exertion of less pressure on the soft tissue mucosa and the underlying bone of the ridge and more force transmission to the implants This can in turn be attributed to the disturbance in local microcirculation of tissues surrounding the bone, thereby altering the mineralization pathway of the bone. ${ }^{[28-30]}$ 
Reduced rate of RRR in the present study may be attributed to the routine follow-up visits to check the fit and occlusion of the denture. These recall visits monitored the bone remodeling which takes one year after prosthesis delivery to follow the shape of the denture-fitting surface, after which the forces may be better distributed with less RRR in the next few years. ${ }^{[31]}$

This result is in contrast with Ahmad et al (2015) ${ }^{[32]}$ who investigated the residual ridge resorption (RRR) induced by an implant-retained overdenture versus conventional complete denture and concluded that implant overdenture resulted in at least twice the RRR as complete denture. This could be caused by the higher hydrostatic stress and less effective energy absorption capabilities of the mucosa underneath the implant overdenture. While implants associated with the implant overdenture provide stronger bite force, they could potentially concentrate hydrostatic stress and cause greater RRR compared to a conventional complete denture.

This contradictory result may be due to the smaller contact area that may cause more localized RRR in implant overdenture retained by only two implants placed in the canine regions unlike this study on four implant assisted overdenture.

When comparing between the two groups, a statistically significant higher ridge resorption was found in group B during the first six months whereas in the second six months, the difference was insignificant. This may be due to the distribution and sharing of load between the implants and the residual ridge in group A (quadrilateral distribution of implant).

This result was consistent with Oh et al (2020) ${ }^{[33]}$ who indicated the significance of additional implant placement in the posterior mandible for preservation of the residual alveolar ridge ,as bone loss in the posterior edentulous mandible was found to be lower when the mandible was restored with 4 IOD rather than with 2 IOD. Although, bone loss in the posterior edentulous mandible was similar when the mandible was rehabilitated with either 2 IOD or CD.

N. Pham et al (2021) ${ }^{[34]}$ conducted a systemic review to clarify the rate of posterior residual ridge resorption (PRRR) in different denture treatments and the factors that can affect PRRR. Meta-analyses revealed that the 4-IOD treatment could lower the rate of PRRR compared with the 2-IOD treatment. However, there was no significant difference in the rate of treatment effect between the CD and 2-IOD treatments.

The same finding was declared by Jacobs et al ${ }^{[35]}$ who demonstrated a minimal posterior mandibular ridge resorption in patients with four implant-supported prostheses. A more considerable posterior ridge resorption was observed in the complete denture wearers.

The study revealed less values of bone resorption in group A compared to group B; this may be due to more stress concentration around tilted implants in group B. This was coping with Hirata et al ${ }^{[36]}$ who declared that the tilted implant was subjected to about twice as much strain as the one perpendicular to the occlusal plane.

In a Finite element analysis study conducted by Yunus et al 2020 ${ }^{[37]}$ to compare between different approaches for the rehabilitation of severe atrophy cases, models of the three implant configurations (Protocol 1: Four anterior implants, Protocol 2: Four anterior and two short posterior implants, Protocol 3: Two anterior and two tilted posterior implants: All-on- $4^{\mathrm{TM}}$ concept) were generated for 10 patients and analyzed under different loading conditions including chewing, biting, and impact forces. Protocol 2 led to the lowest stress concentrations over the mandible among the three protocols. Protocol 2 resulted in significantly lower stresses than Protocol 3 and Protocol 1 over prosthetic bars under chewing forces. In conclusion, Protocol 2 was biomechanically more ideal than Protocol 1 and Protocol 3 to manage the posterior edentulism.

\section{Conclusion:}

From the results of this study, it could be concluded that: 1- In regard to the accepted limits of residual alveolar bone loss, the two design concepts with either 1st molar short implant or premolar distally inclined implant can be used for assisting the mandibular complete overdentures. 2- The posterior short implant concept can be considered more favorable design than inclined implant concept when designing the 4implant assisted mandibular complete overdenture, regarding the preservation of alveolar bone height 


\section{References:}

[1] Cakarer S, Can T, Yaltirik M, Keskin C. Complications associated with the ball, bar and locator attachments for implant-supported overdentures. Med Oral Patol Oral Cir Bucal 2011;16(7):3-10.

[2] Feine, J.S., Carlsson, G.E., Awad, M.A. C, A., Duncan, W.J., Gizani, S., Head, T. H, G., Lund, J.P., MacEntee, M., Mericske-Stern R, Mojon, P., Morais, J.A., Naert, I., Payne AG, Penrod, J., Stoker, G.T., Tawse-Smith, A. T, T.D., Thomason, J.M., Thomson WM\& W, et al. The McGill consensus statement on overdentures. Mandibular two-implant overdentures as first choice standard of care for edentolous patient. 17: 601-602. Gotfredsen, K., Carlsson, G.E., Jokstad, A.,. Int J Oral Maxillofac Implants 2002;17:601-2.

[3] Emami E, Michaud PL, Sallaleh I, Feine JS. Implant-assisted complete prostheses. Periodontol 2000 2014;66(1):119-31.

[4] Oda K, Kanazawa M, Takeshita S, Minakuchi S. Influence of implant number on the movement of mandibular implant overdentures. J Prosthet Dent 2017;117(3):380-5.

[5] Krennmair G, Seemann R, Weinländer M, Piehslinger E. Comparison of ball and telescopic crown attachments in implant- retained mandibular overdentures: a 5-year prospective study. Int J Oral Maxillofac Implant 2011;26(3):598-606.

[6] Warin P, Rungsiyakull P, Rungsiyakull C, Khongkhunthian P. Effects of different numbers of minidental implants on alveolar ridge strain distribution under mandibular implant-retained overdentures. J. Prosthodont. Res.2018;62(1):35-43.

[7] Pimentel MJ, Silva WJ, Del Bel Cury AA. Short implants to support mandibular complete dentures photoelastic analysis. Braz. Oral Res. 2017;31:1-10.

[8] Capelli M, Zuffetti F, Del Fabbro M, Testori T. Immediate rehabilitation of the completely edentulous jaw with fixed prostheses supported by either upright or tilted implants: a multicenter clinical study. Int J Oral Maxillofac Implants 2007;22(4):22.

[9] Annibali S, Cristalli MP, Dell'Aquila D, Bignozzi I, La Monaca G, Pilloni A. Short dental implants: A systematic review. J Dent Res 2012;91(1):25-32.

[10] Kirkpatrick L, Feeney B. A simple guide to IBM SPSS statistics for version 20.0. Student ed. Wadsworth: Cengage Learning; 2013.

[11] Babu B, Jain V, Pruthi G, Mangtani N, Pillai R. Effect of denture soft liner on mandibular ridge resorption in complete denture wearers after 6 and 12 months of denture insertion: A prospective randomized clinical study. J Indian Prosthodont Soc 2017;17:233-8.

[12] Regragui A, Alidrissi N, Sefrioui A, Benfdil F, Merzouk N, Means P. How to Overcome Bone Resorption in Complete Removable Prosthesis ? 2020;7(7):1-5.

[13] Km S, Koli DK, Jain V, Pruthi G, Nanda A. Journal of Oral Biology and Craniofacial Research Comparison of ridge resorption and patient satisfaction in single implant-supported mandibular overdentures with conventional complete dentures: A randomised pilot study. J Oral Biol Craniofacial Res [Internet] 2021;11(1):71-7. Available from: https://doi.org/10.1016/j.jobcr.2020.11.014

[14] Jayaram B, Shenoy KK. Analysis of Mandibular Ridge Resorption in Completely Edentulous Patients Using Digital Panoramic Radiography. IOSR-JDMS 2017;16(8):66-73.

[15] 15. Kordatzis K, Wright PS, Meijer HJ a. Posterior mandibular residual ridge resorption in patients with conventional dentures and implant overdentures. Int J Oral Maxillofac Implants 2003;18(3):447-52.

[16] Wright PS, Glantz P, Watson RM. The effects of fixed and removable implant-stabilised prostheses on posterior mandibular residual ridge resorption. Clin Oral Implant Res 2002;13:169-74.

[17] Yunus N, Saub R, Bai Taiyeb Ali T, Mohd Salleh N RBM. Patient-based and clinical outcomes of implant tele- scopic attachment-retained mandibular overdentures: a 1- year longitudinal prospective study. Int J Oral Maxillofac Implant 2014;29:1149-1156.

[18] Bae J-C, Jeong S-H, Jeong C-M HJ-B. Comparison of the masticatory functions of complete dentures and implant-retained overdentures. Int J Prosthodont 2015;28.

[19] Kremer U, Schindler S, Enkling N, Worni A, Katsoulis J, Mericske-Stern R. Bone resorption in different parts of the mandible in patients restored with an implant overdenture. A retrospective radiographic analysis. Clin Oral Implants Res 2016;27(3):267-72. 
[20] Khalifa AK, Wada M, Ikebe K, Maeda Y. To what extent residual alveolar ridge can be preserved by implant? A systematic review. Int J Implant Dent 2016;2(1):22.

[21] Emera RMK, Abdel-Khalek EA. Five-year radiographic changes of residual ridges under maxillary complete denture and its opposing mandibular two implant-retained overdenture with different resilient attachments. EDJ 2019;65:531-40.

[22] Adina Mosnegutu, Daniel Wismeijer WG. Implant-supported mandibular overdentures can minimize mandibular bone resorption in edentulous patients_ results of a long-term radiologic evaluation PubMed. Int J Oral Maxillofac Implant 2015;30(6):1378-86.

[23] Tian Y, Li Z, Chen J, Yuan X, Sadowsky SJ, Coyac BR, et al. Mechano-adaptive Responses of Alveolar Bone to Implant Hyper-loading in a pre-clinical in vivo model. Clin Oral Implants Res 2020;31(12):1159-72.

[24] Chang M, Chronopoulos V, Mattheos N. Impact of excessive occlusal load on successfullyosseointegrated dental implants: A literature review Impact of excessive occlusal load on successfully- osseointegrated dental implants : a literature review. 2013;(December 2016).

[25] Tymstra N, Raghoebar GM, Vissink A, Meijer HJA. Maxillary anterior and mandibular posterior residual ridge resorption in patients wearing a mandibular implant-retained overdenture. J Oral Rehabil 2011;38(7):509-16.

[26] Utz KH. Studies of changes in occlusion after the insertion of complete dentures ( Part I ). J Oral Rehabil 1996;23:321-9.

[27] Roberts we. Implants: Bone physiology and metabolism. Cda j. 1987;15(2):54-61.

[28] Assad AS, Abd El-Dayem MA, Badawy MM. Comparison between mainly mucosa-supported and combined mucosa-implant-supported mandibular overdentures. Implant Dent 2004;13(4):386-94.

[29] Elsyad M, Habib A. Implant-supported versus implant-retained distal extension mandibular partial overdentures and residual ridge resorption: a 5-year retrospective radiographic study in men. Int $\mathbf{J}$ Prosthodont 2011;24:306-313.

[30] de Jong MH, Wright PS, Meijer HJ, Tymstra N. Posterior mandibular residual ridge resorption in patients with overdentures supported by two or four endosseous implants in a 10-year prospective comparative study. Int J Oral Maxillofac Implants. 2010;25:1168.

[31] Ahmad R. The Relationship of mandibular morphol- ogy with residual ridge resorption associated with im- plant-retained overdentures. Int J Prosthodont 2016;29:573-580.

[32] Ahmad R, Chen J, Abu-hassan MI, Li Q, Swain M V. Resorption Under Implant-Retained Overdentures and Complete Dentures in the Mandible. Int J Oral MaxIllOfac Implant 2015;30:65766.

[33] Oh W, Saglik B, Bak S-Y. Bone Loss in the Posterior Edentulous Mandible with Implant-Supported Overdentures vs Complete Dentures: A Systematic Review and Meta-Analysis. Int J Prosthodont 2020;33(2):184-91.

[34] Pham NQ, Gondab T, Maedab Y, Ikebe K. Average rate of ridge resorption in denture treatment: A Systematic Review. J Prosthodont Res 2021;65:1-9.

[35] Jacobs R, Schotte A, van Steenberghe D, Quirynen M, Naert I. Posterior jaw bone resorption in osseointegrated implant-supported overdentures. Clin Oral Implants Res [Internet] 1992;3(2):63-70. Available from: http://www.ncbi.nlm.nih.gov/pubmed/15900670

[36] Hirata, Kiyotaka; Takahashi, Toshihito; Tomita, Akiko; Gonda, Tomoya; Maeda Y. EBSCOhost 109645170 _ The Influence of Loading Variables on Implant Strain When Supporting DistalExtension Removable Prostheses_An In Vitro Study. Int J Prosthodont 2015;28(5):484-6.

[37] Arslan YZ, Karabulut D, Kahya S, Cansiz E. Biomechanical comparison of implantation approaches for the treatment of mandibular total edentulism. Proc Inst Mech Eng Part $\mathrm{H} J$ Eng Med 2020;234(10):1139-50. 